\title{
EL PROCESO DE PAZ CON LAS FARC: ¿UNA OPORTUNIDAD PARA REDUCIR LA POBREZA RURAL Y AUMENTAR LA PRODUCTIVIDAD AGROPECUARIA?
}

\section{The peace agreement with the FARC: is this an opportunity to reduce rural poverty and increase agricultural productivity?}

\author{
Ana María Ibáñez \\ Profesora Titular, Facultad de Economía, Universidad de los Andes. Contacto: aibanez@uniandes.edu.co
}

\begin{abstract}
Resumen
Más de 50 años de conflicto armado en Colombia han tenido un impacto importante sobre la población y el crecimiento económico del país. Dado que el conflicto armado sucede mayoritariamente en las áreas rurales del país, los efectos económicos del conflicto han sido aún más elevados en estos territorios. El objetivo de este artículo es describir el impacto del conflicto armado sobre la población rural y las condiciones actuales de las áreas rurales así como discutir si el acuerdo de paz con las FARC contribuirá a mejorar estas condiciones.
\end{abstract}

Palabras clave: conflicto armado, crecimiento económico, desarrollo rural, pobreza.

\section{Introducción}

La agenda de paz con las Fuerzas Armadas Revolucionarias de Colombia (FARC), aún en proceso de negociación, incluye un acuerdo denominado "Hacia un nuevo campo colombiano: Reforma Rural Integral". El propósito general del acuerdo agrario es mejorar las condiciones económicas y sociales de las áreas rurales, fortalecer la presencia del Estado en este territorio y dinamizar la producción agropecuaria.

Concentrar la atención en las áreas rurales del país, tras la firma del acuerdo de paz, es importante por

\begin{abstract}
The long-standing conflict in Colombia has inflicted substantial costs on the population and the economic development of the country. Since conflict occurs mainly in the country's rural areas, the economic impacts of violence are higher for these territories. The purpose of this paper is to describe the impact of armed conflict on Colombia's rural population and the current conditions of rural areas as well as discuss whether the peace agreements with FARC may contribute to improve these conditions.
\end{abstract}

Key words: armed conflict, economic development, poverty, rural development.

dos razones: en primer lugar, porque el sector rural se ha beneficiado menos que el sector urbano del crecimiento económico y la reducción de la pobreza, lo que ha generado una ampliación de la brecha urbanorural (algunas regiones exhiben niveles de desarrollo de países de ingreso medio alto, y otras de países de ingreso bajo).

En segundo lugar, el conflicto armado colombiano tuvo como escenario principal el campo, con efectos sociales como el desplazamiento forzado, la destrucción 
de capital como herramienta de incertidumbre, y la constante victimización de la población rural. Esto ha generado unos altísimos costos económicos que retrasan el desarrollo rural y ponen una talanquera a la producción agropecuaria.

La población desplazada, que hoy bordea los seis millones de personas, proviene mayoritariamente de las áreas rurales del país. Más de la mitad de las familias desplazadas poseían tierras como su principal activo de producción. El lucro cesante anual por abandonar la tierra y la producción agropecuaria equivalía, en 2008, al $4.3 \%$ del Producto Interno Bruto Agropecuario - PIB (Ibáñez 2008). Además de estos efectos, el conflicto y la violencia reducen la producción agropecuaria de quienes continúan produciendo pese al conflicto armado. Pinilla (2013) estima que la violencia del conflicto armado reduce el PIB Agropecuario en un 5.8\% anual. En un estudio de pequeños productores agropecuarios, Arias e Ibáñez et al. (2013) encuentran que el conflicto no

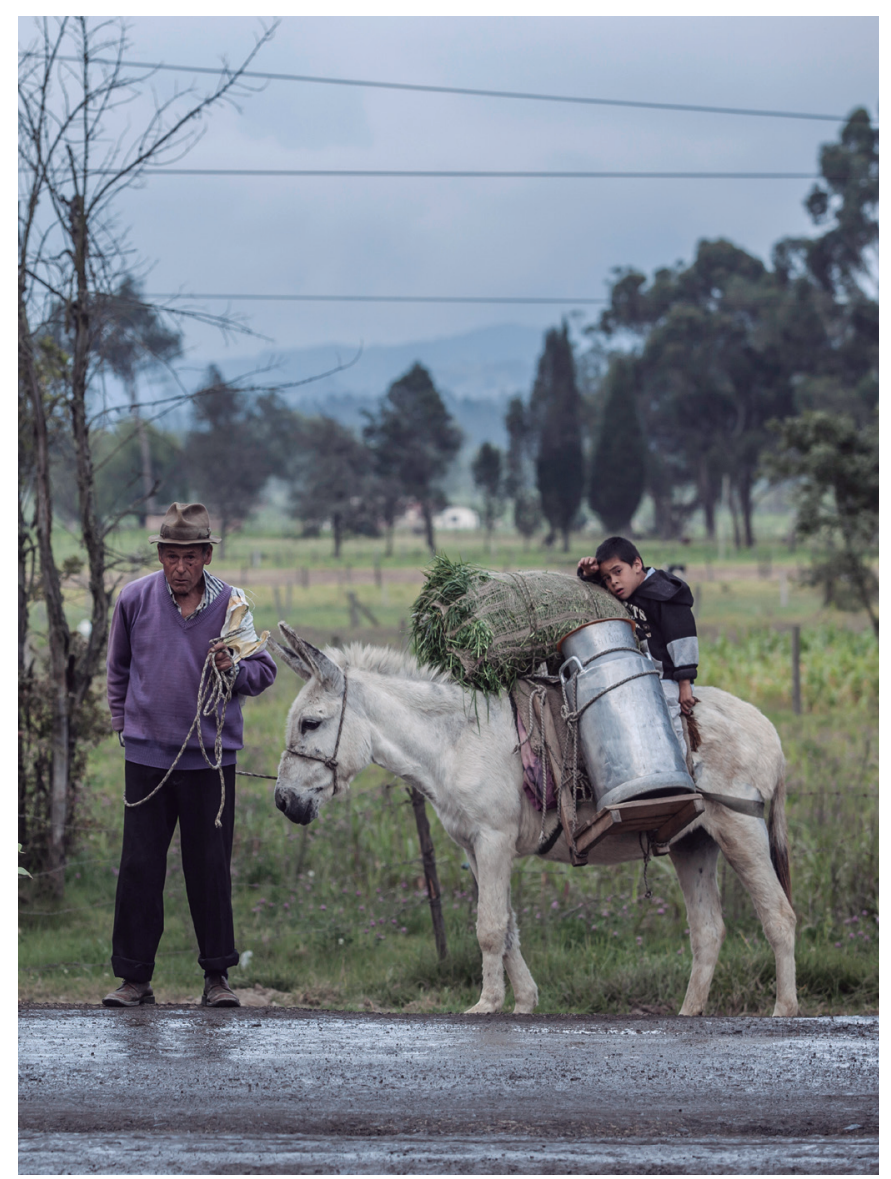

Familia campesina. Simijaca, Boyacá. Fotografía: Alejandro Gómez, Comunicaciones y Marca-ELCA, Uniandes. sólo produce pérdidas por la destrucción, sino por la incertidumbre que conlleva residir en regiones con un conflicto armado activo. Dicha incertidumbre lleva a los productores a concentrarse en cultivos de baja rentabilidad pero bajo riesgo, a reducir el porcentaje de tierra explotada y a elevar la producción ganadera.

El diseño de políticas y su puesta en marcha en las regiones rurales, en especial aquellas que han estado bajo el dominio de los grupos armados ilegales es, por tanto, impostergable. Estos esfuerzos no deben depender de la firma del acuerdo de paz con las FARC. Se deben iniciar pronto. El objetivo de este artículo es describir el acuerdo alcanzado con las FARC y examinar las condiciones actuales de las áreas rurales del país. El artículo introduce, además, este número de la Revista de Ingeniería, cuyo propósito es contribuir con ideas desde la ingeniería para fortalecer el desarrollo del país, así como proponer soluciones prácticas para que el campo sea más productivo, dinámico y con condiciones de vida equitativas.

\section{Hacia un nuevo campo colombiano: Reforma Rural Integral}

Las propuestas del acuerdo agrario apuntan a mejorar las condiciones de la población rural y afianzar la presencia del Estado en estos territorios. Las propuestas se pueden agrupar en tres objetivos: 1) incrementar el acceso a la tierra y proteger los derechos de propiedad; 2) impulsar el desarrollo rural y la producción agropecuaria y 3) reducir la pobreza rural. Además, los acuerdos de participación política y eliminación de cultivos ilícitos incluyen provisiones que complementan el acuerdo agrario a través del fortalecimiento de organizaciones comunitarias, los planes alternativos de sustitución de cultivos y el apoyo a las autoridades municipales, entre otros.

El objetivo de incrementar el acceso a la tierra y proteger los derechos de propiedad se basa en cuatro grandes pilares. Primero, el acuerdo crea un fondo para ampliar el acceso de las familias sin tierra, o tierras insuficientes, a predios que les permitan generar ingresos y salir de la pobreza. Segundo, la titulación de la propiedad y la actualización y formación catastral buscan aumentar la formalidad y la protección de derechos de propiedad. 
Por último, el acuerdo plantea la creación de una jurisdicción agraria necesaria para la solución de disputas por la tierra y la protección de los derechos de propiedad.

Los programas para impulsara la producción agropecuaria, el desarrollo rural y la reducción de la pobreza se pueden agrupar en tres frentes:

1) La delimitación de la frontera agrícola para desestimular la colonización de zonas alejadas de los centros productivos y carentes de Estado.

2) Los estímulos a la producción agropecuaria incluyen programas de asistencia técnica, capacitación, adecuación de tierras, infraestructura, créditos agrarios y recuperación de suelos, entre otros.

3) La provisión de programas sociales -educación, vivienda, salud y agua potable- y de protección social rural son las estrategias propuestas para reducir la pobreza rural.

Las propuestas del acuerdo agrario son en su mayoría necesarias para llevar el Estado a los territorios que han estado ocupados por las FARC durante décadas y a otros territorios rurales. Llevar el estado al territorio, como lo argumenta Claudia López (2016) en esta edición, requiere el fortalecimiento de los gobiernos municipales y departamentales así como de las comunidades. El Estado debe recuperar en estos territorios el "control de la seguridad, la justicia y la tributación, proveer bienes públicos esenciales y ganar una legitimidad duradera en todo el territorio nacional". Sólo de esta manera se evitará que reiniciemos nuevos ciclos de violencia.

López (2016) estima en 455 los municipios -es decir, un $41 \%$ del territorio nacional - que deberán ser priorizados en el acuerdo de paz con las FARC. 172 deberán tener una prioridad alta debido a la presencia histórica de esa guerrilla. 174 una prioridad media debido a la carencia del Estado, la existencia de economías ilegales y la presencia de otros grupos armados, y 109 una prioridad baja debido a su debilidad institucional, alta presencia de víctimas y la existencia de grupos armados ilegales. Estos municipios albergan 15.7 millones de personas y el $\mathbf{7 7 \%}$ de las víctimas. Para alcanzar unas metas ambiciosas de desarrollo en estos territorios, López calcula que se requerirán \$333,5 billones de pesos durante 15 años.

\section{Condiciones de las áreas rurales en Colombia: tierras y producción agropecuaria}

Las condiciones de un alto porcentaje de las áreas rurales del país son precarias debido a la ausencia del Estado, la deficiente provisión de bienes públicos, la baja oferta de programas sociales y la poca conexión con los mercados. Según la Misión para la Transformación del Campo (2014), el 61\% de los municipios del país son áreas rurales ${ }^{1}$ o áreas rurales dispersas ${ }^{2}$ que dependen en un alto porcentaje de la producción agropecuaria.

El acceso a la tierra para los pobladores rurales es bajo. Dos de cada cinco pobladores rurales tienen acceso a la tierra con tamaños promedios de 9.4 hectáreas, con una informalidad de la propiedad de casi el 50 \% (Gáfaro, Ibáñez et al. 2014). La propiedad de la tierra en Colombia es, además, altamente concentrada. Según el Tercer Censo Nacional Agropecuario (CNA) (DANE 2014), en Colombia existen 2,7 millones de productores, de los cuales 724.000 residen en sus predios. Un 65\% de los productores residentes se concentran en predios menores a cinco hectáreas, y el 1\% de los propietarios más grandes posee el $42.6 \%$ de las hectáreas rurales (DANE 2014; Gáfaro, Ibáñez et al. 2014).

La sub-explotación de la tierra, por su parte, es alta. Sólo un 38.6\% de la tierra rural (43.1 millones de hectáreas) está destinada al uso agropecuario. La explotación se concentra mayoritariamente en la ganadería extensiva (79,7\%), mientras la producción agrícola ocupa apenas un $20.1 \%$, lo cual equivale a 8.6 millones de hectáreas.

Los productores tradicionales cosechan cinco millones de hectáreas de la producción agrícola, mientras que los agroindustriales cultivan 3.6 millones. Si bien la propiedad está concentrada en grandes productores, la productividad agrícola de los pequeños y medianos pareciera ser más alta: un 40 \% del área cosechada está en predios de menos de 50 hectáreas, y estos predios generan más del $40 \%$ de la producción agropecuaria (DANE 2014).

1 Cabeceras pequeñas con densidades entre 10 y 100 habitantes por kilómetro cuadrado. 2 Cabeceras pequeñas con densidades menores a 10 habitantes por kilómetro cuadrado. 
Además de la violencia y la excesiva concentración de la tierra, el escaso acceso a insumos y bienes públicos productivos, así como el atraso tecnológico, contribuyen a la poca explotación de la tierra y baja productividad agropecuaria. Un $16.8 \%$ de los productores agropecuarios reportan contar con construcciones productivas, y un $16.6 \%$ con maquinaria productiva. Al respecto, Ospina (2016) señala que el uso de maquinaria aumenta en un $13 \%$ la productividad de los pequeños productores. Sin embargo, el acceso a ella está concentrado en los productores de más de 1.000 hectáreas, de los cuales un 51.1\% reporta tenerla. Solo el $11.4 \%$ de los propietarios de menos de cinco hectáreas cuentan con maquinaria productiva.

Por otra parte, el acceso al crédito también es escaso: $11.1 \%$ de los pequeños productores solicitan financiación y $90 \%$ de estos la consiguen (DANE 2014).

La innovación tecnológica en la producción agropecuaria y en las áreas rurales es baja o casi nula. Según el Tercer Censo Nacional Agropecuario, un 10\% de los productores recibe asistencia técnica, lo cual inhibe la adopción de nuevas tecnologías productivas. Por ejemplo, Camacho and Connover (2010) muestran que el uso de celulares para recibir información de precios agrícolas mejora las condiciones de los productores agrícolas al reducir la dispersión en el precio de venta de sus productos y disminuir la pérdida de su cosecha entre el $11 \%$ y el $14 \%$.

Pese a la importancia de la tecnología móvil, muchas regiones del país aún no reciben señal o tienen un acceso deficiente y débil a internet. La baja rentabilidad para el sector privado de llevar estas tecnologías a zonas rurales aisladas determina este bajo acceso (Balen y Valenzuela, 2016). Balen y Valenzuela (2016) propone en esta edición de la Revista diseñar soluciones creativas para ampliar la cobertura de las Tecnologías de la Información en áreas rurales, tales como permitir que pequeños operadores regionales usen los denominados 'espacios blancos', que no están siendo utilizados por los grandes operadores por considerarlos de escasa rentabilidad; así mismo, promover que los operadores compartan infraestructura en zonas rurales no rentables y proveer incentivos tributarios para la compra de teléfonos móviles inteligentes, similares a aquellos dados para compra de computadores.
El aislamiento de los mercados al que muchos productores rurales se ven sometidos debido a la deficiencia de las vías, tiene un gran impacto sobre la competitividad y bienestar de las familias rurales. Carlos F. Sánchez (2016) discute en este número, basado en datos de la Encuesta Longitudinal de la Universidad de los Andes, cómo las intervenciones viales en áreas rurales aumentan la productividad agrícola, abren nuevos mercados, aumentan la especialización de los productores agrícolas, el consumo agregado de los hogares y la participación de éstos en actividades no agrícolas, ayudando a reducir la pobreza en dos puntos porcentuales.

Pese a la importancia de las vías secundarias y terciarias para conectar a los agricultores con los mercados en donde comercializan sus productos, las condiciones de éstas son deficientes. En este número, Germán Ospina (2016) hace un importante diagnóstico de la red vial en Colombia, que cubre cerca de 216.000 km, de los cuales el $8 \%$ hace parte de la red primaria, operada por entidades del Gobierno Nacional (como el Invías); el $21 \%$ a la red secundaria, a cargo de los gobiernos departamentales, y el $71 \%$ restante corresponde a la red terciaria, administrada por el Gobierno Nacional y los entes territoriales.

Después de la transferencia del manejo de vías que hizo la Nación a las gobernaciones y municipios, en 1993, poco se conoce sobre longitud y condiciones exactas. Además, las especificaciones y calidad de las vías son heterogéneas a lo largo del territorio nacional. Ospina (2016) argumenta que existen recursos suficientes para ampliar y mejorar las redes secundarias y terciarias del país, así como la posibilidad del uso de tecnologías menos costosas para hacerlo. Por ejemplo, en algunas vías terciarias la pavimentación no es la mejor alternativa, pues existen tecnologías más adecuadas y menos costosas. Por último, el autor recomienda que se deben diseñar alternativas para promover la participación de la población en la construcción y mantenimiento de las vías.

Por otra parte, la dificultad para acceder al agua, tanto los hogares como para la producción agrícola, es un problema latente y sin soluciones tangibles en el país rural. Según el CNA (2013), un 93.6\% de los productores tienen acceso al agua, pero de estos más de la mitad 


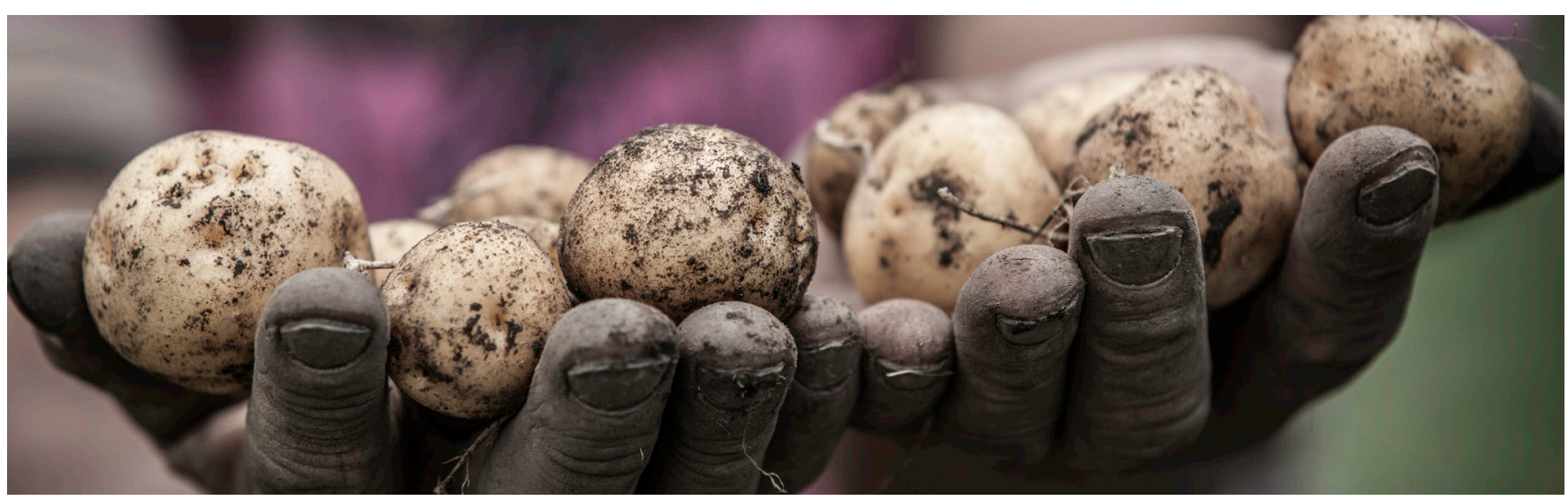

Cosecha. Fotografía: Alejandro Gómez, Comunicaciones y Marca-ELCA, Uniandes.

(54.2\%) reportan tener dificultades para el acceso. Para analizar a fondo este problema, esta edición cuenta con una entrevista realizada a Carlos Angulo, ex rector de la Universidad de los Andes, quien analiza la importancia de los distritos de riego en Colombia y los retos que éstos conllevan.

Según Angulo, al garantizar la continuidad del suministro de agua en épocas de escasez, un distrito de riego da predictibilidad a los agricultores acerca de la oferta de agua, aumenta el número de cosechas al año y reduce el riesgo del daño de cosechas. Sin embargo, es enfático en que la construcción de distritos de riego es el primer paso, y que esta debe estar acompañada de asistencia técnica para que los productores adopten mejores tecnologías y aprovechen la tierra y el suministro en su máximo potencial. Afirma que se debe evitar el error cometido en proyectos de distritos de riego anteriores, en los cuales no se proveía esta asistencia. Angulo propone hacer un inventario de los distritos de riego del país para saber cuántos funcionan en la actualidad y cómo se puede expandir su capacidad. Así mismo, propone identificar las zonas con potencial para iniciar la construcción de nuevos distritos.

A lo anterior se suma la deficiente provisión de acueducto y alcantarillado en las áreas rurales del país. William Carrasco (2016) enseña un panorama desolador: la cobertura de acueducto y alcantarillado en las áreas rurales es de $73 \%$ y $68 \%$ respectivamente, mientras estas cifras son 97\% y 93\% para las áreas urbanas. Los cubrimientos rurales están muy por debajo de lo establecido en los Objetivos del Milenio, lo cual implica que, aunque Colombia cumplió con este objetivo a nivel nacional, en las áreas rurales no lo hizo. Además de la escasa provisión de acueducto y alcantarillado, la calidad del agua que llega a los hogares rurales, según el Îndice de Riesgo de Calidad del Agua (IRCA), es mala. Mientras el promedio de riesgo fue bajo para las áreas urbanas del país, para las áreas rurales fue significativo.

Carrasco (2016) argumenta que la baja provisión del acueducto y alcantarillado es resultado de un mal diseño institucional. Su recuento histórico revela cómo a lo largo de las décadas se ha desmontado el aparato institucional que garantizaba la construcción de redes para la provisión de acueducto y alcantarillado. Este diseño institucional permitía una mayor participación de las organizaciones comunitarias y garantizaba la financiación por parte del Gobierno Nacional y las autoridades municipales y departamentales. Para mejorar la provisión en las áreas rurales, Carrasco (2016) propone modificar el diseño institucional, de tal manera que le permita a las comunidades participar en la oferta, mejorar el control sobre los prestadores, fortalecer el sistema de información y contar con tecnologías apropiadas para prestar el servicio en las áreas rurales.

\section{Conclusiones}

Las condiciones de vida de la población rural y la precariedad de los servicios a los que pueden acceder los productores agropecuarios impiden que el país aproveche su capacidad productiva. El acceso a la tierra, principal activo productivo de los pobladores rurales, 
es bajo. La propiedad está altamente concentrada; la cobertura de bienes públicos productivos es escasa, y muy pocos productores agropecuarios cuentan con insumos esenciales para aumentar su productividad. Además, muchas regiones rurales están aisladas de los centros productivos y de los mercados debido a la precariedad vial del país. Por último, el conflicto armado y la violencia han estado presentes en el país rural durante muchas décadas, dificultando mucho más la presencia real del Estado y la provisión de servicios públicos eficientes.

No es una sorpresa, por tanto, la alta pobreza rural y el pobre desempeño de la producción agropecuaria. Si bien la pobreza rural ha disminuido, la caída ha sido más lenta que los índices nacionales: en 1997 el índice de pobreza rural era de $87 \%$ y el urbano $50 \%$; en 2014 estas cifras eran $44 \%$ y $15 \%$ respectivamente. El crecimiento del PIB agropecuario ha estado consistentemente por debajo del PIB nacional. Según la Misión de Transformación del Campo, entre 2003 y 2007 el PIB Nacional creció a un $5.9 \%$ y el agropecuario un 3\%, y estas cifras para 2007 y 2013 eran del $4 \%$ y el $1.5 \%$ respectivamente.

El acuerdo agrario es una buena excusa para consolidar la presencia del Estado en las áreas rurales del país, expandir la oferta de servicios sociales y bienes públicos productivos y llevar los mercados a las regiones. El acuerdo agrario consigna propuestas para lograr parte de este objetivo. Aunque no se avalara el acuerdo entre el Gobierno y las FARC, muchas de las propuestas consignadas en ese documento se deben poner en marcha con vigor y altas inversiones. Otorgar más oportunidades económicas a la población rural y romper el ciclo de violencia no sólo beneficiará a estas regiones sino a todo el país.

\section{Referencias}

Arias, M. A., A. M. Ibáñez, et al. (2013). Agricultural Production amidst Conflict: The Effects of Shocks, Uncertainty and Governance of Non-State Armed Actors, Universidad de los Andes.

Balen y Valenzuela (2016). "Las tecnologías de la información y las comunicaciones en el posconflicto colombiano", Revista de Ingeniería.
Camacho, A. and E. Connover (2010). The Impact of Receiving Price and Climate Information in the Agricultural Sector. F. d. Economia. bogotá, Colombia, Universidad de los Andes.

DANE (2014). Tercer Censo Nacional Agropecuario.

Gáfaro, M., A. M. Ibáñez, et al. (2014). Equidad y eficiencia rural en Colombia: una discusión de políticas para el acceso a la tierra. Equidad y movilidad social: Diagnósticos y propuestas para la transformación de la sociedad colombiana. M. Melendez and A. Montenegro. Bogotá, Ediciones Uniandes.

Ibáñez, A. M. (2008). El desplazamiento forzoso en Colombia: un camino sin retorno hacia la pobreza Bogotá, Ediciones Uniandes.

López, Claudia (2016). "Aprender de nuestra experiencia para que la paz sí le cumpla esta vez a los colombianos", Revista de Ingeniería.

Ospina, Germán (2016). "Las carreteras secundarias y los caminos vecinales en el desarrollo de Colombia", Revista de Ingeniería.

Ospina, S. (2016). Eficiencia y tamaño del predio agrícola en Colombia. Facultad de Economía. Bogota, Colombia, Universidad de los Andes. Tesis de Maestría.

Sánchez, Carlos Felipe (2016). "Colombia en el postcuerdo y el rol de la infraestructura de transporte. ¿Hacia dónde van las vías?" Revista de Ingeniería, edición 44. Universidad de los Andes. 